\title{
A new signal injection method with PSO for multi-carrier predistortion
}

\author{
Xiaofang $\mathrm{Wu}^{1 \mathrm{a})}$, Wei Wang ${ }^{1}$, Yun $\mathrm{Xu}{ }^{1}$, and Jiangnan Yuan ${ }^{2}$ \\ ${ }^{1}$ School of Information Science and Technology, Xiamen University, \\ Xiamen 361005, China \\ ${ }^{2}$ School of Photoelectricity and Communication Engineering, Xiamen University of \\ Technology, Xiamen 361005, China \\ a)xfwu@xmu.edu.cn
}

\begin{abstract}
In multi-carrier transmitters, nonlinear characteristic of power amplifiers (PAs) results in intra-band distortion and interband distortion. The intra-band distortion exists in most singlecarrier transmitters as well, and can be removed by traditional digital predistortion (DPD) techniques. However, the inter-band distortion is rather unique for the multi-carrier linearization. A channel-selective multi-cell DPD architecture which deals with the intra-band and inter-band distortion separately is used in this paper. Furthermore, a new signal injection method with particle swarm optimization (PSO) is proposed to suppress the inter-band distortion in the paper. The injected inter-band signals are firstly reconstructed by a memory polynomial DPD, and then their amplitude and phase are fine-tuned by a PSO algorithm. Simulation results show that the method restrains inter-band distortion significantly and accelerates the convergence speed of PSO algorithm.
\end{abstract}

Keywords: signal injection, particle swarm optimization, power amplifier, digital predistortion

Classification: Microwave and millimeter wave devices, circuits, and systems

\section{References}

[1] Y. Liu, W. Chen, J. Zhou, B. Zhou and F. M. Ghannouchi: IEEE Trans. Microw. Theory Tech. 61 [1] (2013) 281.

[2] S. A. Bassam, M. Helaoui and F. M. Ghannouchi: IEEE Trans. Commun. 60 [8] (2012) 2344.

[3] F. M. Ghannouchi and O. Hammi: IEEE Microw. Mag. 10 [7] (2009) 52.

[4] A. Singh, J. E. Scharer, J. H. Booske and J. G. Wohlbier: IEEE Trans. Electron Devices 52 [5] (2005) 709.

[5] S. Chen: IEEE Trans. Broadcast. 57 [4] (2011) 856.

[6] P. L. Carro, P. G. Ducar, J. de Mingo and A. Valdovinos: 16th IST Mobile and Wireless Communications Summit (2007) 1.

[7] L. Ding, G. T. Zhou, D. R. Morgan, Z. Ma, J. S. Kenney, J. Kim and C. R. Giardina: IEEE Trans. Commun. 52 [1] (2004) 159.

[8] L. Li, J. Feng, S. Cai and X. Liang: IEEE Vacuum Electronics Conference (2008) 105. 


\section{Introduction}

In order to improve the spectrum efficiency, multi-carrier transmission is widely used in modern communication systems. However, these multicarrier transmitting signals are envelope-varying and with high peak-toaverage power ratios (PAPRs). In order to meet the linearity requirements, they usually lead to the sacrifice of systems' power efficiency. Moreover, it should be noticed that the nonlinear behavior in multi-carrier transmitters is quite different from that in the single-carrier systems [1]. The multicarrier transmission not only cause the fundamental intermodulation distortion (IMD) entitled intra-band distortion but also the cross-band IMD distortion entitled inter-band distortion [2].

Since the traditional digital predistortion (DPD) techniques [3] designed for the single band distortion just compensate for the intra-band distortion, they cannot provide satisfactory performance for multi-carrier transmitters. Similarly, signal injection [4] has been proved to be another valid method to suppress the IMD, but most of the related works published in prior literatures are limited in the analog domain and merely aim at removing the intra-band distortion. Aidin et al. [2] firstly introduced the signal injection method to restrain the inter-band distortion in the digital domain, but the two injecting IMD signals are fixed with equal magnitude and $180^{\circ}$ degree phase shift compared to the inter-band IMD product terms, so that it is difficult to ensure the inter-band distortion of the transmitter can be well mitigated when nonlinear device and circuit parameters change. In other words, in order to obtain better effect, the amplitude and phase of the two injecting signals should be adjusted simultaneously.

Particle swarm optimization (PSO) is an efficient population-based stochastic global optimization technique inspired by social behavior of bird flocks or fish schools, and it has been successfully applied to DPD model optimization applications, which enjoys a low computational cost compared with mathematical derivation [5]. Recently, Chen [5] built a DPD model by PSO. However, when the precision of the model is increasing, the dimension that PSO required is double increasing. The rate of convergence slows down significantly. P. L. et al. [6] used PSO to calculate the coefficients of the DPD direct learning structure, where the dimension of PSO was 16 and the iteration number of convergence was about 1600 . So the PSO method will get into convergence trouble when the dimension of DPD model is large.

In this work, a signal injection method controlled by PSO is proposed to restrain the inter-band distortion. To our knowledge, it is the first time that PSO algorithm is used for signal injecting method. The proposed model has the following advantages: 1) Both the amplitude and phase of the two injecting signals are adjusted by PSO method, so it is feasible for different PA circuits when compared with [2]; 2) The dimension of PSO for the signal injection method is only 4 so that the rate of convergence is fast when compared with $[5,6] ; 3)$ No extra auxiliary equipment is needed to extract the third order IMD (IMD3) products when compared with analog signal injection method.

The organization of this paper is as follows. Section 2 describes a channel-selective multi-cell DPD structure. The proposed method of signal 
injection with PSO is presented in Section 3. Section 4 shows simulation results and Section 5 concludes the paper.

\section{The multi-cell processing DPD structure}

A channel-selective multi-cell processing predistortion (MCP-DPD) structure [2] is used in this paper, which is developed to suppress both the intraband distortion and the inter-band distortion in different channels. An outstanding benefit of the MCP-DPD structure is that it can reduce the sampling rate significantly compared with the traditional single-band DPD. The MCP-DPD is composed of two major processing approaches. The first one aims at compensating for intra-band distortion in the transmission bands with center carrier frequencies $f_{1}$ and $f_{2}$; and the second one aims at canceling the IMD products in the inter-band with center IMD3 frequencies $2 f_{1}-f_{2}$ and $2 f_{2}-f_{1}$. Fig. 1 shows the block diagram of the multi-cell processing architecture. It consists of three major stages: the signal extraction and analysis stage, 2) the processing stage, 3) the synthesizing stage. The processing cells $\mathrm{C} 2$ and $\mathrm{C} 3$ are developed to compensate for the intra-band nonlinearities in the B2 and B3 bands, while cells C1 and C4 are developed to eliminate the inter-band distortions in the B1 and B4 bands.

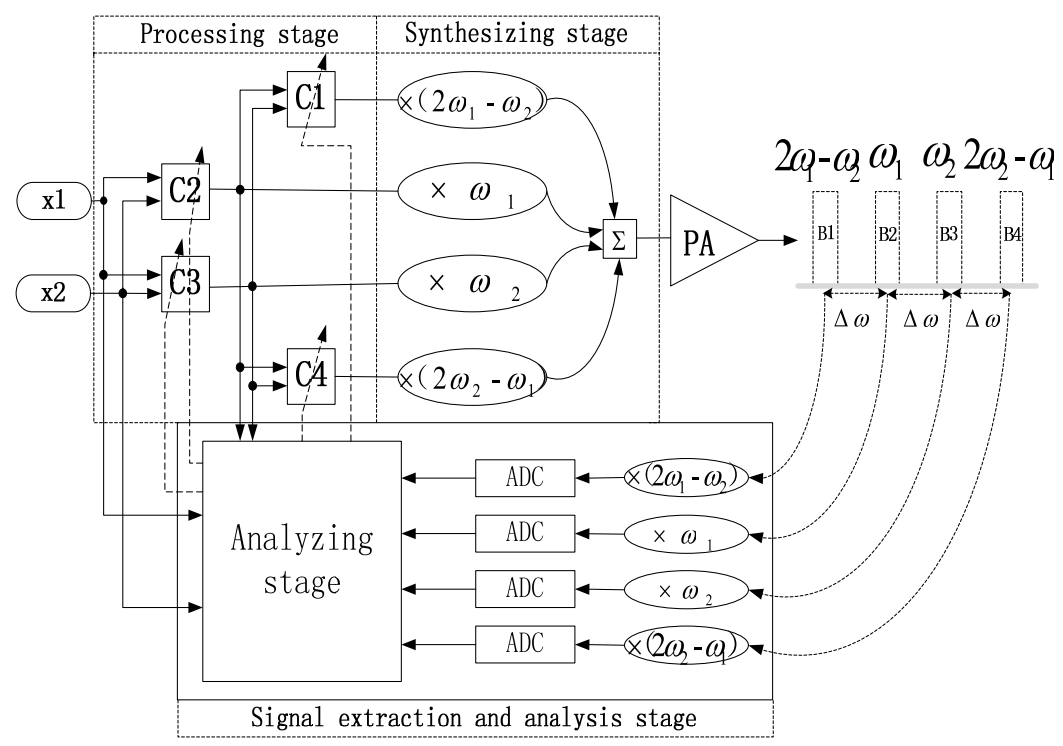

Fig. 1. MCP-DPD [2]

The intra-band predistortion model including the processing cells $\mathrm{C} 2$ and C3 uses Knoecker product model [2]. The generalized model for the nonlinear system with N-order nonlinearities and (M-1)-order memory depth is as follows:

$$
\begin{aligned}
& y^{i}(n)=\sum_{p=0}^{M-1} \vec{c}_{1, p}^{i} \vec{x}_{p}+\vec{c}_{2, p}^{i}\left(\vec{x}_{p} \otimes\left|\vec{x}_{p}\right|\right)+\ldots \\
& +\vec{c}^{i}{ }_{N, p}\left\{\vec{x}_{p} \otimes\left(\left|\vec{x}_{p}\right| \otimes\left|\vec{x}_{p}\right| \otimes \ldots \otimes\left|\vec{x}_{p}\right|\right)\right\} ; i=1,2
\end{aligned}
$$

where $\vec{c}_{n, p}^{i}$ are the coefficients of the DPD model, $\vec{x}_{p}=\left[\begin{array}{l}x_{1}(n-p) \\ x_{2}(n-p)\end{array}\right]$ is the 
$p$ th-delayed vector of the two-carrier complex baseband signals. $\otimes$ means Kronecker product of two vectors. $\left|\vec{x}_{p}\right|$ is the element-wise operator to calculate the absolute value of each elements of the vector $\vec{x}_{p} \cdot y^{i}(n)$ is the output of the $i$ th processing cell $(i=2,3)$.

To build Kronecker model, we must calculate the coefficients $\vec{c}_{n, p}^{i}, n=1,2, \cdots, N$ by the analysis stage, where the coefficients are determined by least square method. Using the Kronecker model, it is feasible to compensate for intra-band distortions in the transmitting band B1and B2. The in-depth analysis can be referred to [2].

\section{The signal injection method with PSO for inter-band DPD}

In order to suppress the inter-band distortion, signal injection method controlled by PSO is proposed in this paper. Firstly inter-band IMD3 products are reconstructed by a memory polynomial (MP) DPD [7], and then PSO is used to search for the best amplitude and phase of the interband IMD3 injecting signals. At last, inject them into the PA to restrain inter-band distortion.

Fig. 2 is the flow chart of the inter-band DPD processing stage, i.e., the cell $\mathrm{C} 1$ and $\mathrm{C} 4$ in Fig. 1. The output of PA is filtered by band-pass filter centered at $2 f_{1}-f_{2}$ (corresponding to $\mathrm{C} 1$ ) or $2 f_{2}-f_{1}$ (corresponding to C4), and down-converted to baseband. The obtained baseband-version inter-band distortion is used as the expected output of inter-band MP model, and the output of intra-band DPD (i.e., the cell C2 and C3 in Fig. 1) is used as the input of inter-band MP model. Thus depending on the fitting polynomial coefficients and the input of MP model, we can reconstruct the inter-band distortion without repetitive measurement and operation at the output port of PA. The reconstructed signals is used as the embryo of injecting inter-band signals, and the PSO algorithm is used to search for the perfect amplitude and phase of injecting signals. At last, up-convert the injecting signals to B1 and B4 bands respectively and combine them with the up-converted intra-band DPD's output signals. The combined signal of processing stage is sent to the PA to compensate for the inter-band distortion and the intra-band distortion as well.

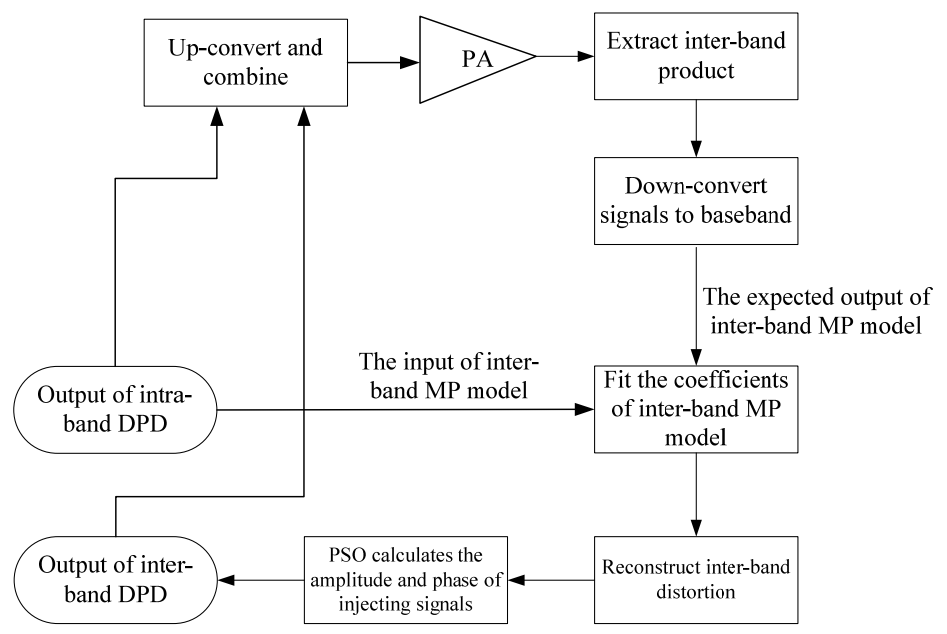

Fig. 2. Flow chart of inter-band DPD processing stage 
The output signal of inter-band MP DPD model can be written as:

$$
\vec{y}_{I M P}=L_{\vec{x}}^{F} \vec{h}
$$

where $\vec{y}_{I M P}=\left[y_{I M P}(1) \cdots y_{I M P}(L)\right]^{T}$ is an $L \times 1$ vector representing $L$ samples of the inter-band distortion product in baseband version, and $\vec{h}=\left[h_{1} \cdots h_{N}\right]^{T}$ is the $N \times 1$ vector of the polynomial coefficients. $L_{\vec{x}}^{F}=L P F\left\langle L_{\vec{x}} \times e^{-j \omega_{I M P} t}\right\rangle$ is the complex baseband low-pass filtered signal of the matrix $L_{\vec{x}}$, and $\omega_{I M P}=2 \pi f_{I M P}$ is the center angular frequency of the inter-band.

$L_{\vec{x}}=\left[\begin{array}{ccc}x(1) & x(1)|x(1)| & x(1)|x(1)|^{N-1} \\ \vdots & \ldots & \vdots \\ \vdots & \ddots & \vdots \\ \vdots & \ldots & \vdots \\ x(L) & x(L)|x(L)| & x(L)|x(L)|^{N-1}\end{array}\right]$ is a $L \times N$ matrix, which is

built based on the complex input signal $x(l)$, and $\vec{x}=[x(1) \cdots x(L)]^{T}$ is an $L \times 1$ vector representing $L$ samples of the input signal, i.e., the output of the intra-band DPD.

The polynomial coefficient vector, $\vec{h}$, is derived from the pseudoinversing of the $L_{\vec{x}}^{F}$ matrix as follows:

$$
\vec{h}=\operatorname{pinv}\left(L_{\vec{x}}^{F}\right) \vec{y}_{I M P}
$$

Then we can reconstruct the inter-band product terms $\vec{x}_{c}$ in each interband as

$$
\vec{x}_{c}=\vec{L}_{x} \vec{h}
$$

where $\vec{x}_{c}=\left[x_{c}(1) \cdots x_{c}(L)\right]^{T}$ is an $L \times 1$ vector. Due to the PA's nonlinear behavior, we need to calculate the amplitude scaling and phase shift of the two injecting inter-band signals on the basis of $\vec{x}_{c}$. Assuming the amplitude scaling and phase shift of the injecting signals in B1 and B4 band to be $\gamma_{1}$, $\varphi_{1}$ and $\gamma_{4}, \varphi_{4}$ respectively, and regarding them as the four dimensions of PSO, the actual injecting signals are as follows:

$$
\begin{aligned}
& \vec{y}_{\text {inject } 1}=\gamma_{1} \times \vec{x}_{c(1)} \times e^{j 2 \pi \varphi_{1}} \\
& \vec{y}_{\text {inject } 4}=\gamma_{4} \times \vec{x}_{c(4)} \times e^{j 2 \pi \varphi_{4}}
\end{aligned}
$$

where $\vec{x}_{c(1)}$ and $\vec{x}_{c(4)}$ are $\vec{x}_{c}$ in B1 and B4 band respectively; besides, $\vec{y}_{\text {inject } 1}$ and $\vec{y}_{\text {inject } 4}$ are the injecting signals in B1 and B4 band respectively.

The power of $L$ samples of inter-band product terms from PA's output $\varepsilon=\sum_{n=1}^{L}\left|e_{i n t e r}(n)\right|^{2}$ is used as the fitness function of PSO. When $L$ is large enough, $\varepsilon$ can present the power of inter-band distortion. The less power is, the better effect of restraining inter-band distortion we can get. By calculating the minimum of $\varepsilon$, we can get $\gamma_{1}, \varphi_{1}, \gamma_{4}$ and $\varphi_{4}$ of the injecting signals.

The steps of the PSO algorithm are as follows:

1) Initialize the position and velocity of each particle randomly. The position and velocity vector of the $i$ th particle are $\mathrm{X}^{i}=\left(x_{i, 1} x_{i, 2} x_{i, 3} x_{i, 4}\right)$ and $V^{i}=\left(v_{i, 1} v_{i, 2} v_{i, 3} v_{i, 4}\right)$ respectively. The 
four dimensions in each vector correspond to $\gamma_{1}, \varphi_{1}, \gamma_{4}$ and $\varphi_{4}$.

2) Evaluate the fitness $\left(\varepsilon=\sum_{n=1}^{L}\left|e_{\text {inter }}(n)\right|^{2}\right)$ of each particle. Store the current position and fitness of each particle into the pbest which is the optimal solution of each particle. Store the position and fitness of the best one among pbest into the gbest which is the optimal solution of the entire group.

3) Update the particle's velocity and position by (6).

$$
\begin{aligned}
& v_{i, j}(t+1)=w v_{i, j}(t)+c_{1} r_{1}\left[p_{i, j}-x_{i, j}(t)\right]+c_{2} r_{2}\left[p_{g, j}-x_{i, j}(t)\right] \\
& x_{i, j}(t+1)=x_{i, j}(t)+v_{i, j}(t+1), j=1,2, \cdots, d
\end{aligned}
$$

where $w$ is the inertia weight factor, $c_{1}$ and $c_{2}$ are positive learning factors, $r_{1}$ and $r_{2}$ are random numbers with uniform distribution between 0 and 1 .

4) Compare the current fitness value of each particle with its best position ever experienced. If the current fitness is smaller, store the current position and fitness into pbest. Compare all the current pbest and gbest, and then update gbest.

5) If match the stop condition (usually the fitness value precision or default iterations), stop searching and output the result. Otherwise return to 3 ).

\section{Simulation results}

The performance of the proposed method is examined through MATLAB with four-carrier (1001) WCDMA signal, which is collected by a signal collector. The PSO parameters are set as: particle swarm size $=30$, $c_{1}=c_{2}=2.05$, and $w=0.7$. In order to accelerate the convergence speed, we delimit the initial amplitude and phase range of the particle: both $\gamma_{1}$ and $\gamma_{4}$ are between 0.1 and 3 , both $\varphi_{1}$ and $\varphi_{4}$ are between 0 and $2 \pi$. A popular memory polynomial PA model [7] extracted from an actual Class AB PA is used in the simulation, and the relation of its output $y(n)$ and input $x(n)$ is defined by:

$$
y(n)=\sum_{k=1, k \in \text { odd }}^{K} \sum_{m=0}^{M-1} a_{k m} x(n-m)|x(n-m)|^{k}
$$

where

$$
\begin{aligned}
& a_{10}=1.0513+0.0904 j, a_{11}=-0.0680-0.0023 j, a_{12}=0.0289-0.0054 j \\
& a_{30}=-0.0542-0.2900 j, a_{31}=0.2234+0.2317 j, a_{32}=-0.0621-0.0932 j . \\
& a_{50}=-0.9657-0.7028 j, a_{51}=-0.2451-0.3735 j, a_{52}=0.1229+0.1508 j
\end{aligned}
$$

Fig. 3 shows the power spectral density (PSD) of the output signal from PA. The blue line represents the original WCDMA input signal. The purple line represents the output signal without DPD. The intra-band and interband distortion without DPD is clear to see. The carrier-to-intermodulation-products power ratio (CIMPR) [2] has come to nearly $24 \mathrm{dBc}$. The black line represents the output signal only operating intra-band DPD. The green line represents the output signal with both intra-band and inter-band 
DPD. By PSO algorithm, we can obtain that the optimal amplitude and phase of the two injecting signals is $\gamma_{1}=1.3986, \varphi_{1}=203.544^{\circ}, \gamma_{4}=0.4846$, and $\varphi_{4}=50.292^{\circ}$. The figure shows that with the intra-band DPD, the adjacent-channel power ratio (ACPR) of the intra-band distortion has been suppressed almost $14 \mathrm{dBc}$. Since the intra-band DPD filters out a little inter-band distortion, the CIMPR also has been improved about $10 \mathrm{dBc}$. With both the intra-band and the proposed inter-band DPD, the CIMPR has been further improved about $19 \mathrm{dBc}$ and comes to nearly $45 \mathrm{dBc}$. Although the injecting method makes the ACPR performance slightly worse (about $3 \mathrm{dBc}$ ), both the inter-band and intra-band power spectrum are restrained to below $-40 \mathrm{dBc}$, overall the proposed method can effectively compensate for the nonlinear distortion of multi-carrier PA.

According to [2], the inter-band distortion can be well suppressed as long as inverting the phase of inter-band IMD $180^{\circ}$. However, according to

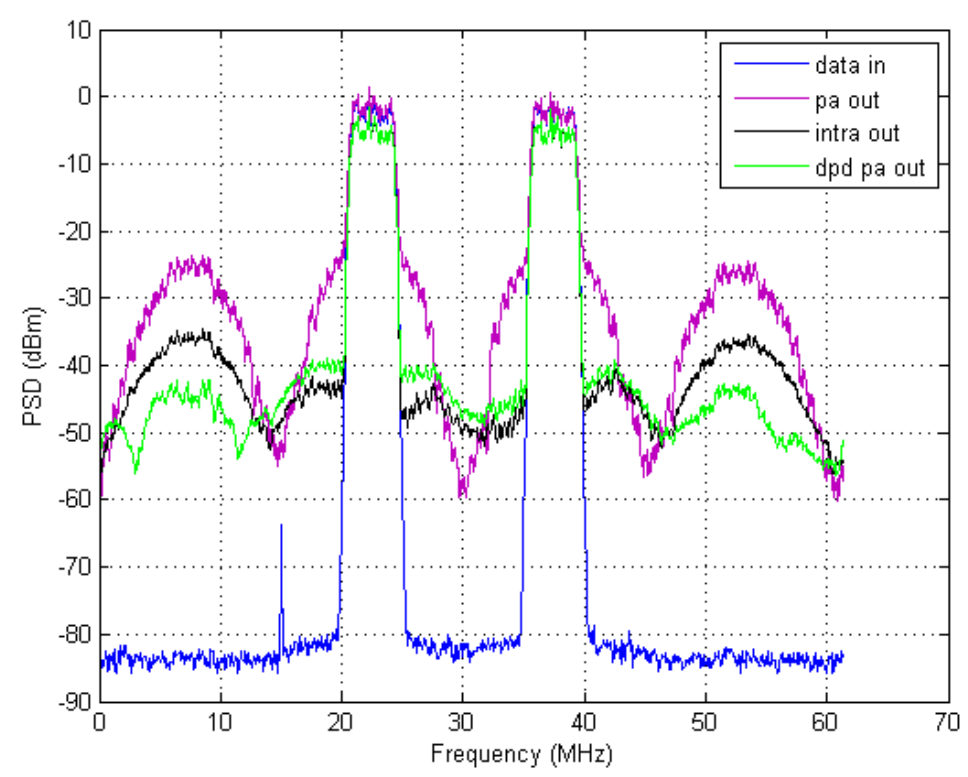

Fig. 3. Power spectral density of PA output

[8], the best effect is attained with about $45^{\circ}$ phase change of IMD by experiment, this means that the optimal phase is not fixed and is related to a certain PA. Furthermore, the amplitude is also an important role of the injecting signals.

Fig. 4 demonstrates the comparison of PSD with different signal injection methods. The purple line represents PA output without DPD. The blue line represents the output after using the signal injecting method with phase changing $180^{\circ}$ as [2], the cyan one represents the output after changing $45^{\circ}$ as [8], the black one represents the output after changing $225^{\circ}$, and the green one represents the output after the signal injection method with PSO. Table I shows the comparison of the ACPR at frequency offsets of $5 \mathrm{MHz}$ and the CIMPR in inter-bands. We can see from Fig. 4 and Table I that the proposed signal injection method can improve the CIMPR about $19 \mathrm{dBc}$ compared with no DPD, and improve the CIMPR about $5 \mathrm{dBc}$ better than the second best injecting signal with phase changing $225^{\circ}$; and about $10 \mathrm{dBc}$ better than [2]. Overall, the proposed method can greatly improve the ability of restraining the inter-band 
distortion.

Fig. 5 shows the AM-AM curve and the AM-PM curve of the PA. The purple points represent the signal without DPD and the green points

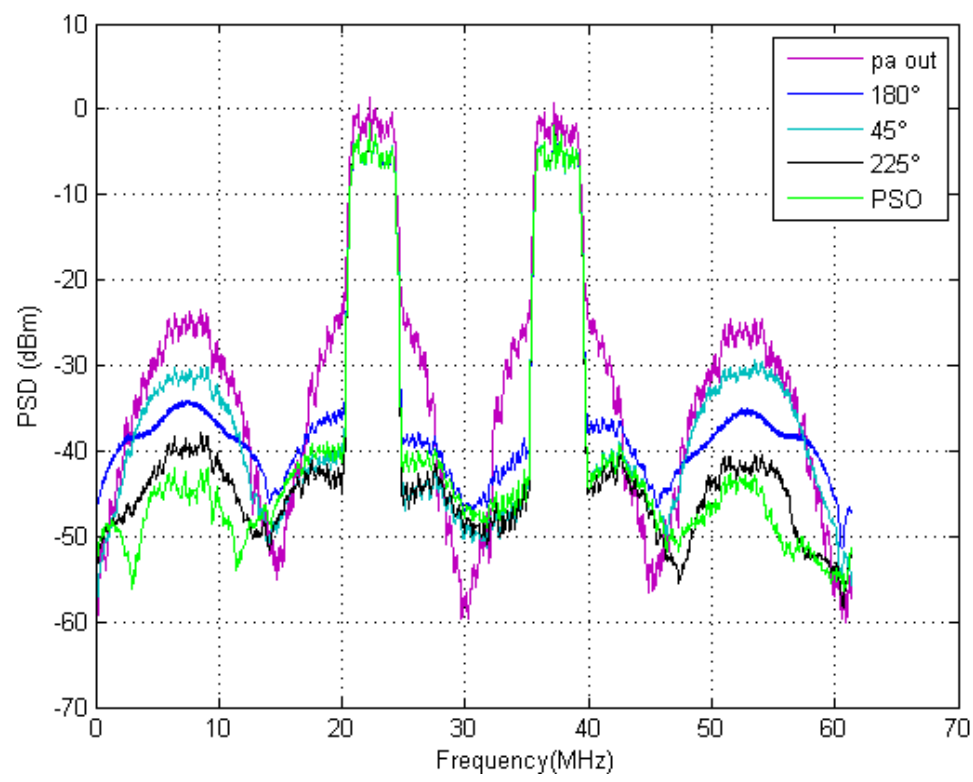

Fig. 4. Comparison of PSD with the signal injection methods

Table I. Comparison of ACPR and CIMPR

\begin{tabular}{|c|c|c|c|c|}
\hline \multirow{2}{*}{ Scenario } & \multicolumn{2}{|c|}{ ACPR(dBc) offsets of 5MHz } & \multicolumn{2}{c|}{ CIMPR(dBc) } \\
\cline { 2 - 5 } & Left & Right & Left & Right \\
\hline Without DPD & -34.5 & -32.36 & 25.74 & 27.01 \\
\hline $180^{\circ}[2]$ & -38.39 & -41.76 & 34.45 & 35.59 \\
\hline $45^{\circ}[8]$ & -41.7 & -46.6 & 31.37 & 30.84 \\
\hline $225^{\circ}$ & -41.7 & -46.07 & 39.65 & 41.98 \\
\hline PSO & -40.57 & -45.07 & 45.2 & 43.57 \\
\hline
\end{tabular}

represent the signal with the proposed DPD. It is obviously that DPD can compensate for the nonlinear characteristic of the PA significantly.

Fig. 6 shows the iterative convergence curve of the PSO algorithm. Since the dimension of the particle is only 4 , the PSO algorithm converges
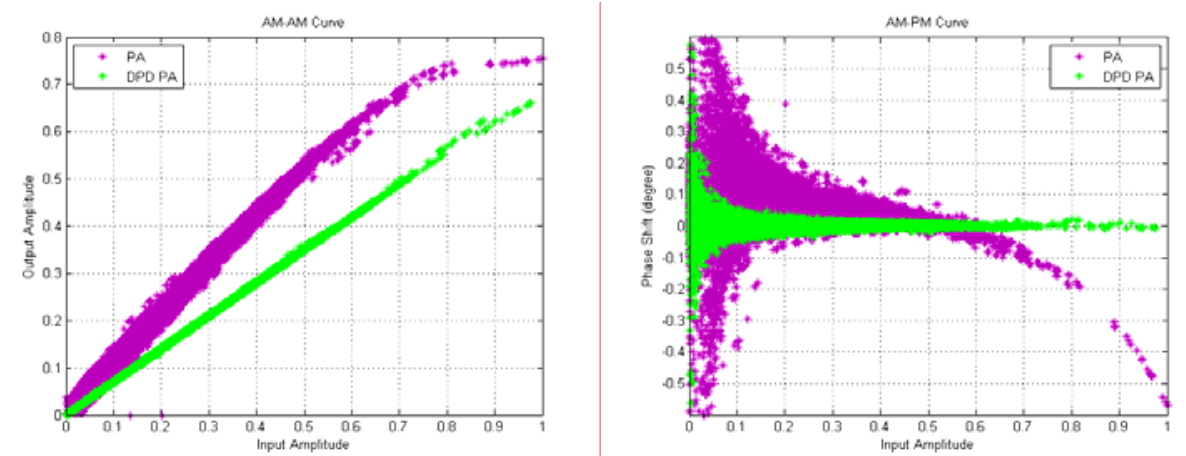

Fig. 5. Normalized AM-AM and AM-PM curves 
quickly, and the fitness value is steady around $1.5 \times 10^{-5}$ after 25 iterations.

\section{Conclusion}

A channel-selective multi-cell DPD structure is used to compensate for the

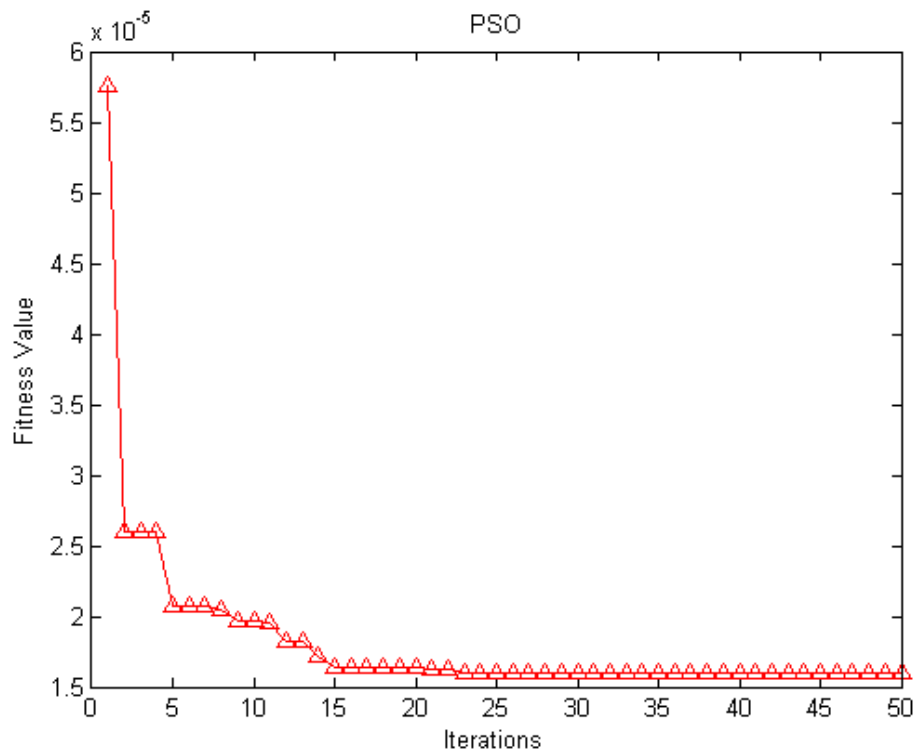

Fig. 6. PSO Iterative Convergence Curve

nonlinear distortion of multi-carrier PAs, which rectifies the intra-band and inter-band distortion in different channels. For inter-band DPD, a new signal injection method with PSO is proposed, where an intelligent PSO algorithm is introduced to calculate the amplitude scaling and phase shift of the injecting inter-band intermodulation signals. In general, this method increases the ability of restraining the inter-band distortion and has a fast convergence speed. From the simulation results, the proposed method can obtain the ACPR improvement of about $12 \mathrm{dBc}$ and the CIMPR improvement of about $19 \mathrm{dBc}$ respectively. 